\title{
Acute exacerbations in the INPULSIS trials of nintedanib in idiopathic pulmonary fibrosis
}

\author{
Harold R. Collard ${ }^{1}$, Luca Richeldi ${ }^{2,3}$, Dong Soon Kim4, Hiroyuki Taniguchi ${ }^{5}$, \\ Inga Tschoepe ${ }^{6}$, Maurizio Luisetti ${ }^{+7}$, Jesse Roman ${ }^{8}$, Gregory Tino ${ }^{9}$, \\ Rozsa Schlenker-Herceg ${ }^{10}$, Christoph Hallmann ${ }^{11}$ and Roland M. du Bois ${ }^{12}$
}

Affiliations: ${ }^{1}$ Division of Pulmonary and Critical Care Medicine, Dept of Medicine, University of California San Francisco, San Francisco, CA, USA. ${ }^{2}$ National Institute for Health Research Southampton Respiratory Biomedical Research Unit and Clinical and Experimental Sciences, University of Southampton, Southampton, UK. ${ }^{3}$ Division of Respiratory Medicine, Università Cattolica del Sacro Cuore, Fondazione Policlinico “A. Gemelli", Rome, Italy. ${ }^{4}$ Asan Medical Center, University of Ulsan, Seoul, South Korea. ${ }^{5}$ Dept of Respiratory Medicine and Allergy, Tosei General Hospital, Aichi, Japan. ${ }^{6}$ PRA Health Sciences, Paris, France. ${ }^{7}$ University of Pavia, Pavia, Italy. ${ }^{8}$ University of Louisville School of Medicine and Robley Rex VA Medical Center, Louisville, KY, USA. ${ }^{9}$ Penn Presbyterian Medical Center, Dept of Medicine, Philadelphia, PA, USA. ${ }^{10}$ Boehringer Ingelheim Pharmaceuticals Inc., Ridgefield, CT, USA. ${ }^{11}$ Boehringer Ingelheim Pharma GmbH \& Co. KG, Ingelheim am Rhein, Germany. ${ }^{12}$ Imperial College, London, UK.

Correspondence: Harold R. Collard, Division of Pulmonary and Critical Care Medicine, Dept of Medicine, University of California San Francisco, 505 Parnassus Avenue, San Francisco, CA, 94131, USA.

E-mail: Hal.CollardQucsf.edu

@ERSpublications

Investigator-reported and confirmed/suspected acute exacerbations of IPF have similar risk factors and outcomes http://ow.ly/k1Sj309SZSf

Cite this article as: Collard HR, Richeldi L, Kim DS, et al. Acute exacerbations in the INPULSIS trials of nintedanib in idiopathic pulmonary fibrosis. Eur Respir J 2017; 49: 1601339 [https://doi.org/10.1183/ 13993003.01339-2016].

ABSTRACT Time to first investigator-reported acute exacerbation was a key secondary end-point in the INPULSIS trials of nintedanib in patients with idiopathic pulmonary fibrosis (IPF).

We used the INPULSIS trial data to investigate risk factors for acute exacerbation of IPF and to explore the impact of nintedanib on risk and outcome of investigator-reported and adjudicated confirmed/ suspected acute exacerbations. Mortality following these events and events adjudicated as not acute exacerbations was analysed using the log rank test.

Risk of acute exacerbations was most strongly associated with the following variables: baseline forced vital capacity (higher risk with lower value), baseline supplemental oxygen (higher risk with use), baseline antacid medication (higher risk with use), treatment (higher risk with placebo), and for confirmed/ suspected acute exacerbations, cigarette smoking. Mortality was similar following investigator-reported and adjudicated confirmed/suspected acute exacerbations. Nintedanib had no significant effect on risk of mortality post-exacerbation.

Investigator-reported acute exacerbations of IPF are associated with similar risk factors and outcomes as adjudicated confirmed/suspected acute exacerbations.

This article has supplementary material available from erj.ersjournals.com

Earn CME accreditation by answering questions about this article. You will find these at erj.ersjournals.com/journal/cme Received: July 062016 | Accepted after revision: Feb 092017

The INPULSIS trials are registered at www.clinicaltrials.gov with identifiers NCT01335464 and NCT01335477.

Support statement: The INPULSIS trials were funded by Boehringer Ingelheim. Funding information for this article has been deposited with the Crossref Funder Registry.

Conflict of interest: Disclosures can be found alongside this article at erj.ersjournals.com

Copyright OERS 2017 


\section{Introduction}

Idiopathic pulmonary fibrosis (IPF) is a progressive disease characterised by worsening dyspnoea and loss of lung function [1]. While some patients with IPF experience a steady decline in lung function, others experience sudden acute deteriorations after a period of relative stability [2]. A perspective published by an international group of experts in 2007 defined acute exacerbations of IPF as acute deteriorations in symptoms associated with changes in radiographic appearance and the absence of an identifiable cause [3]. Published incidence rates for acute exacerbations of IPF vary, owing to differences in case definition, study methodology and the population studied, but they are believed to occur in approximately 5-10\% of patients annually [1].

Low vital capacity/forced vital capacity (FVC) has been shown to be a reproducible risk factor for acute exacerbations of IPF [4-8]. Other suggested risk factors include worse dyspnoea [4, 5]; worse gas exchange [7]; honeycombing and greater extent of disease on high-resolution computed tomography (HRCT) [9]; gastroesophageal reflux disease [10, 11]; elevated serum KL-6 [12]; and air pollution [13]. Acute exacerbations are associated with high morbidity and high short-term mortality $[5,6,14]$.

Nintedanib is an intracellular inhibitor of tyrosine kinases, including the receptors for fibroblast growth factor, platelet-derived growth factor and vascular endothelial growth factor [15], and has been approved for the treatment of IPF in several countries and regions including the US, EU and Japan. In the Phase II TOMORROW trial, nintedanib $150 \mathrm{mg}$ twice daily was associated with a significantly reduced incidence of investigator-reported acute exacerbations (defined using similar criteria to the perspective published in 2007 [3]) compared with placebo over 52 weeks [16]. These results were not reliably reproduced in the Phase III INPULSIS trials, as there was a significant difference in favour of nintedanib for time to first acute exacerbation observed in INPULSIS-2 (hazard ratio (HR) $0.38 ; 95 \%$ CI $0.19,0.77 ; \mathrm{p}=0.005$ ), but no significant difference between the nintedanib and placebo groups observed in INPULSIS-1 (HR 1.15; 95\% CI $0.54,2.42 ; \mathrm{p}=0.67$ ) [17]. In pooled data from the INPULSIS trials, a pre-specified sensitivity analysis of events centrally adjudicated as confirmed or suspected acute exacerbations demonstrated a $68 \%$ reduction in the risk of an adjudicated confirmed or suspected acute exacerbation with nintedanib therapy [17].

In this analysis, we used the INPULSIS clinical trial data to further investigate the risk factors for acute exacerbation of IPF, and to explore the impact of nintedanib on risk and outcome of investigator-reported and confirmed/suspected acute exacerbations.

\section{Methods}

Trial design

The current analyses utilised data from the INPULSIS trials (NCT01335464 and NCT01335477), the design of which has been described previously [17]. Briefly, eligible patients had a diagnosis of IPF confirmed by central review of an HRCT scan (and surgical lung biopsy, if available), FVC $\geqslant 50 \%$ predicted, diffusing capacity of the lung for carbon monoxide (DLCO) 30-79\% predicted and forced expiratory volume in $1 \mathrm{~s}\left(\mathrm{FEV}_{1}\right) / \mathrm{FVC}$ ratio $\geqslant 0.7$. Patients were randomised $3: 2$ to receive nintedanib $150 \mathrm{mg}$ twice daily or placebo for 52 weeks, followed by a 4 -week follow-up period. Treatment interruption and dose reduction from $150 \mathrm{mg}$ twice daily to $100 \mathrm{mg}$ twice daily were allowed for the management of adverse events. The protocol for the INPULSIS trials was approved by an ethics committee or institutional review board at every participating centre, and informed consent was obtained from all participants. For the current analyses, the corresponding author had access to all the data and analyses, and had final responsibility for the decision to submit the results for publication.

\section{Adjudication of acute exacerbations}

An acute exacerbation was defined in the INPULSIS trial protocol as an event meeting all of the following criteria: unexplained worsening or development of dyspnoea within 30 days; new diffuse pulmonary infiltrates on chest radiograph and/or HRCT parenchymal abnormalities with no pneumothorax or pleural effusion (new ground-glass opacities) since the previous visit; and possible causes of the acute worsening, including infection, left heart failure, pulmonary embolism or any identifiable cause of acute lung injury, excluded by routine clinical examination and microbiological studies. In cases of an adverse event that was considered by an investigator to fulfil the criteria for an acute exacerbation, the investigator was requested to complete an Acute Exacerbation Questionnaire (supplementary material) and to provide supporting documentation, to the extent available, for use in the adjudication process.

The adjudication committee comprised three experts in IPF (M. Luisetti, J. Roman, G. Tino) who were not investigators in the INPULSIS trials. Members of the committee noted their assessment of every event sent for adjudication on an Acute Exacerbation Adjudication Worksheet (supplementary material) prior to the committee meeting. These worksheets were used to facilitate the discussion and were not collected. At the time of adjudication, an Adjudication Form (supplementary material) was completed by the committee. An event 
was adjudicated as a "confirmed acute exacerbation" if all the protocol-defined criteria were met, a "suspected acute exacerbation" if the event was felt to be an acute exacerbation but failed to meet all protocol-specified criteria, or "not an acute exacerbation" if an alternative cause was identified. The committee adjudicated events according to their collective medical judgement; there was no defined algorithm. The committee was blinded to treatment allocation, and events were adjudicated before database lock.

\section{Statistical analyses}

All analyses were performed on pooled data from the INPULSIS trials. A risk factor analysis was conducted to construct Cox proportional hazard models of the time to first investigator-reported exacerbation over 52 weeks and time to first adjudicated confirmed or suspected acute exacerbation over 52 weeks [6]. Risk factor candidates were classified by treatment group (nintedanib versus placebo), race (Asian versus non-Asian) and the following baseline characteristics: FVC \% predicted, DLCO \% predicted, smoking status (former/current smokers versus never-smokers), emphysema on HRCT (yes versus no), diagnostic criteria (honeycombing on HRCT and/or confirmation of usual interstitial pneumonia by surgical lung biopsy versus no honeycombing or biopsy), corticosteroid use (yes versus no), antacid medication (proton pump or histamine receptor-2 inhibitor) use (yes versus no) and supplemental oxygen use (yes versus no). The risk factor candidates were added consecutively into the statistical model (forward stepwise selection), selecting at each step the covariate achieving the smallest p-value in the likelihood chi-squared test. The stepwise selection was stopped when no further covariate achieved a p-value of $<0.2$. The Akaike information criterion (AIC) was calculated to assess the goodness of fit of the statistical models. Hazard ratios and $95 \%$ Wald confidence intervals were calculated for each covariate that was selected in the final model. C-statistics of the final models for investigator-reported and adjudicated confirmed or suspected acute exacerbations were calculated. Owing to geographical variability in season, the seasonality of acute exacerbations was explored by counting events reported in the northern hemisphere in 3-month intervals only.

For the analysis of time to death since onset of first acute exacerbation end-points (i.e. first investigator-reported acute exacerbation, first confirmed or suspected acute exacerbation, or first event adjudicated as not an acute exacerbation), time to event information was used to produce Kaplan-Meier plots for each treatment group and was analysed primarily using the log rank test, with hazard ratios and confidence intervals obtained using the Cox proportional hazards model. All deaths that occurred between randomisation and the end of the post-treatment follow-up period were taken into account in this analysis. Rate of death at fixed intervals after an event (30, 90 and 180 days) was also described.

All analyses were exploratory and were defined post-hoc. All analyses were performed using SAS version $9 \cdot 4$ (SAS Institute, Cary, NC, USA).

\section{Results}

In total, 87 events were adjudicated by the central committee (figure 1). For eight of these events, the investigator sent information to the adjudication committee for review but ultimately did not consider the event to be an acute exacerbation; these events were not included in the 79 investigator-reported acute exacerbations. Of the 79 investigator-reported acute exacerbations, 37 events in 34 patients were in the nintedanib group (5.3\%) and 42 events in 35 patients were in the placebo group (8.3\%). The incidence rates of investigator-reported acute exacerbations in the nintedanib and placebo groups were 5.3 and 8.2 per 100 patient-years, respectively (risk ratio $0.65 ; 95 \%$ CI $0.40,1.04 ; \mathrm{p}=0.07$ ).

Nine investigator-reported acute exacerbations were adjudicated as confirmed acute exacerbations, 33 as suspected acute exacerbations and 35 as not acute exacerbations. For two events, insufficient data were available for adjudication. In both of these cases, however, the patient had already experienced a confirmed or suspected acute exacerbation. Confirmed or suspected acute exacerbations were reported in $2.2 \%$ of patients in the nintedanib group and $5.9 \%$ of patients in the placebo group. The incidence rate of confirmed or suspected acute exacerbations was 2.2 and 5.8 per 100 patient-years in the nintedanib and placebo groups, respectively (risk ratio $0.37 ; 95 \%$ CI 0.19, 0.72; $\mathrm{p}=0.003$ ).

Baseline characteristics of patients who had or did not have one or more investigator-reported acute exacerbations are shown in table 1 (and split by treatment group in supplementary table E1). There were numerical differences between these groups in the proportion of patients who were men, were former or current smokers, had emphysema, or were taking antacid medication or supplemental oxygen, and in mean FVC ( $\mathrm{mL}$ and \% predicted), mean DLCO (\% predicted) and mean St George's Respiratory Questionnaire (SGRQ) total score. The top performing model for risk of investigator-reported acute exacerbation included four variables: FVC at baseline (higher risk with lower value), supplemental oxygen at baseline (higher risk with use), antacid medication at baseline (higher risk with use) and treatment arm (higher risk with placebo) (table 2). The c-statistic for this model was 0.68. 


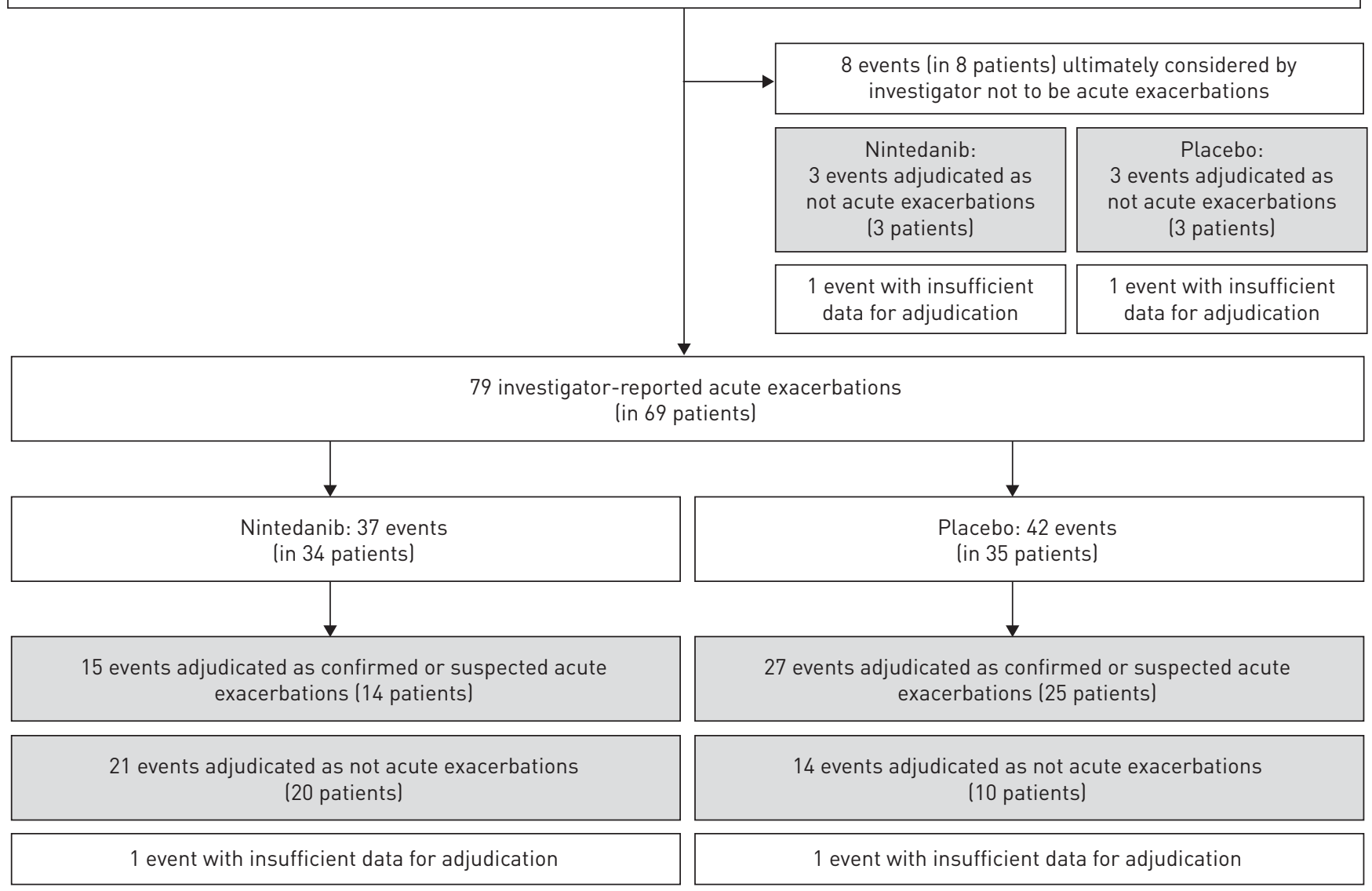

FIGURE 1 Events included in these analyses. Shaded boxes indicate events that were included in the analyses of adjudicated events. This figure indicates only the types of event included in these analyses, not the timing at which investigators and the adjudication committee decided whether an event was an acute exacerbation.

Baseline characteristics of patients who had one or more events adjudicated as a confirmed or suspected acute exacerbation versus patients who had one or more events all adjudicated as not an acute exacerbation are shown in supplementary table E2. The top performing model for risk of confirmed or suspected acute exacerbation included the same four variables as the model for investigator-reported acute exacerbation plus being a former/current smoker (higher risk with history of smoking) (table 2). The c-statistic for this model was 0.71 . Both investigator-reported acute exacerbations and adjudicated confirmed or suspected acute exacerbations appeared to be more common in winter than in other seasons (figure 2).

Mortality was similar following all three types of events (table 3). Nintedanib had no significant impact on the risk of mortality following investigator-reported acute exacerbations, confirmed or suspected acute exacerbations, and events adjudicated as not acute exacerbations, respectively (supplementary figure E1). Importantly, these analyses were uncorrected for other potential determinants of post-acute exacerbation mortality (e.g. FVC, DLCO) as these data were not collected.

\section{Discussion}

Accurate identification of patients at risk for acute exacerbation of IPF will be essential for cohort enrichment strategies in future clinical trials evaluating therapies that aim to reduce acute exacerbation rates. Our findings confirm that acute exacerbations are more likely to occur in patients with more physiologically advanced disease. Specifically, lower FVC \% predicted and the use of supplemental oxygen were associated with an increased risk of acute exacerbation. Other variables identified by our analysis as associated with an increased risk of acute exacerbation included antacid medication use at baseline (a finding that contradicts findings from the IPFnet trials [11] and that is unexplained), treatment with placebo rather than nintedanib and, for adjudicated confirmed or suspected acute exacerbations, current or former smoking (a finding that contradicts previous cohort-derived findings [5]). Acute exacerbations seemed more common in the winter months, suggesting that viral respiratory infection, air pollution or 
TABLE 1 Baseline characteristics of patients who had or did not have at least one investigator-reported acute exacerbation

Patients with investigator-reported acute exacerbation
Patients with no investigator-reported acute exacerbation

\begin{tabular}{|c|c|c|}
\hline Patients & 69 & 992 \\
\hline Age years & $69.1 \pm 7.3$ & $66.6 \pm 8.1$ \\
\hline Male & $58(84.1 \%)$ & $783(78.9 \%)$ \\
\hline \multicolumn{3}{|l|}{ Race } \\
\hline Other (white/black/missing ${ }^{\#}$ ) & $49(71.0 \%)$ & $690(69.6 \%)$ \\
\hline Weight kg & $76.7 \pm 17.8$ & $79.2 \pm 16.5$ \\
\hline Ex- or current smoker & $53(76.8 \%)$ & $712(71.8 \%)$ \\
\hline Bronchodilators & $11(15.9 \%)$ & $190(19.2 \%)$ \\
\hline Proton pump or histamine receptor-2 inhibitor & $33(47.8 \%)$ & $373(37.6 \%)$ \\
\hline Supplemental oxygen & $16(23.2 \%)$ & $76(7.7 \%)$ \\
\hline Emphysema" & $22(31.9 \%)$ & $398(40.1 \%)$ \\
\hline Honeycombing on HRCT & $39(56.5 \%)$ & $528(53.2 \%)$ \\
\hline FVC $\mathrm{mL}$ & $2303 \pm 617$ & $2748 \pm 780$ \\
\hline \multicolumn{3}{|c|}{$\begin{array}{l}\text { Data are presented as mean } \pm S D \text {, unless otherwise indicated. HRCT: high-resolution computed tomography; FVC: forced vital capacity; FEV1: } \\
\text { forced expiratory volume in } 1 \mathrm{~s} \text {; DLCO: diffusing capacity of the lung for carbon monoxide; SGRQ: St George's Respiratory Questionnaire. } \\
\text { Patients were categorised into subgroups depending on whether, between randomisation and the end of the post-treatment follow-up period, } \\
\text { they experienced or did not experience an investigator-reported acute exacerbation. " } \text { : in France, regulations do not permit the collection of } \\
\text { data on race; "ी: based on qualitative assessment of HRCT scans. }\end{array}$} \\
\hline
\end{tabular}

other seasonal factors may contribute to risk. Together, these findings largely reinforce the concept of acute exacerbation of IPF as a clinical event triggered by external injury or stress to the lung, which is more likely to manifest in patients with physiologically advanced disease.

Novel analyses presented in this report demonstrate no significant difference in outcomes between investigator-reported and adjudicated confirmed/suspected acute exacerbations. There was no significant impact of nintedanib treatment on mortality following acute exacerbation and other forms of acute respiratory worsening. While there was a numerical trend in favour of nintedanib treatment, this result needs to be interpreted with caution as the number of events in this analysis was relatively small.

TABLE 2 Risk prediction models for investigator-reported and adjudicated confirmed or suspected acute exacerbations Step

\begin{tabular}{llll}
\multicolumn{2}{c}{ Risk factor analysis } & $\begin{array}{c}\text { Final model } \\
\text { Model variable }^{\#}\end{array}$ & $\begin{array}{l}\text { p-value } \\
\text { HR }\left(95 \% \mathrm{Cl}^{\S}\right)^{\S}\end{array}$
\end{tabular}

Investigator-reported acute exacerbations

Adjudicated confirmed or suspected acute exacerbations

$\begin{array}{cccc}\text { FVC \% predicted at baseline } & <0.0001 & 830.217 & 0.67(0.55-0.80) \\ \text { Supplemental oxygen use at baseline } & 0.0018 & 824.565 & 2.47(1.37-4.47) \\ \text { Antacid medication use at baseline } & 0.0873 & 823.706 & 1.50(0.91-2.47) \\ \quad \text { Randomisation to nintedanib } & 0.1150 & 823.271 & 0.66(0.40-1.08) \\ & & & \\ & 0.0006 & 483.723 & 0.67(0.53-0.86) \\ \text { FVC \% predicted at baseline } & 0.0010 & 475.169 & 0.33(0.16-0.66) \\ \quad \text { Randomisation to nintedanib } & 0.0676 & 473.938 & 1.78(0.92-3.43) \\ \text { Antacid medication use at baseline } & 0.0938 & 472.805 & 2.13(0.89-5.13) \\ \quad \text { Former or current smoker } & 0.1322 & 472.870 & 1.85(0.80-4.29)\end{array}$

FVC: forced vital capacity. ${ }^{\#}$ : covariates that achieved $p<0.2$ in the stepwise selection procedure are shown. The variables are shown in the order that they were selected into the model. ": $p$-value of the likelihood chi-squared test used in the decision to add the variable to the model. ${ }^{+}$: Akaike information criterion (AIC) was calculated for the model at the end of each selection step. Lower AIC values indicate better performance of the model. $\S_{\text {: }}$ based on the final model, i.e. including all the variables that were selected during the stepwise selection procedure. ${ }^{f}$ : hazard ratio $(95 \% \mathrm{Cl})$ is calculated for FVC $\%$ predicted $10 \%$ higher at baseline. 
FIGURE 2 Seasonality of acute exacerbations. Northern hemisphere only. Three investigator-reported acute exacerbations were reported in the southern hemisphere. Some patients had more than one acute exacerbation during the trials.
Investigator-reported acute exacerbations

Adjudicated confirmed/suspected acute exacerbations

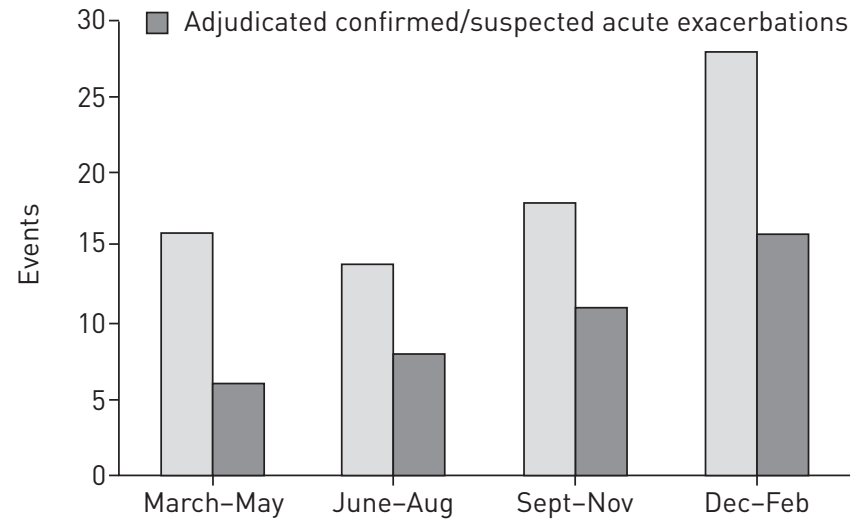

It has been suggested that East Asian patients may be at greater risk of acute exacerbations compared with patients of other races $[18,19]$, but this was not observed in our data. Our findings are consistent with the results of a large Japanese phase III trial of pirfenidone [20], which found a rate of acute exacerbations in line with contemporary trials performed over $48-60$ weeks predominantly in western countries $[6,16,21,22]$. Although there may be important genetic and environmental factors in East Asian patients that contribute to IPF pathobiology, high-quality data from several large datasets suggest that reports of increased risk for acute exacerbation probably result from ascertainment bias (i.e. bias in recognition, reporting and/or classification of events).

Accurate identification of acute exacerbations in clinical trials remains a challenge, as the clinical data required for accurate adjudication are difficult to obtain. Data from the three IPFnet trials showed that of 88 investigator-reported acute exacerbations assessed by an adjudication committee, 31 (35\%) were unclassifiable (based on a similar definition of acute exacerbations to that proposed in 2007 [3]) because of missing data [22]. The concept of suspected acute exacerbations, proposed as clinically suspected acute exacerbations that do not meet all criteria owing to missing data [3], is one approach to overcoming this issue that is supported by our findings. Clinical researchers will need to contextualise the strengths and limitations of differing approaches to event classification within the goals of their study design.

It has recently been proposed by an international working group of experts that the definition of acute exacerbations be revised to remove the requirement for the deterioration in respiratory function to be idiopathic, and to include events triggered by infections, aspiration or other identified factors [23]. Our data support this concept, showing that investigator-reported acute exacerbations, adjudicated confirmed or suspected acute exacerbations and events adjudicated as not acute exacerbations were associated with similar mortality rates. Whether similarities in behaviour are enough to warrant a unified classification or whether aetiological differences remain that could dictate differences in treatment approach remains an important area for future debate.

These data from the INPULSIS trials represent the largest prospective dataset published to date on acute exacerbations in patients with IPF. However, imitations to these data remain, including the exclusion of patients with severe physiological impairment from the INPULSIS trials (i.e. reduced generalisability), the relatively small number of events available for the risk factor analysis and incomplete information available for the adjudication of some events. Stepwise selection procedures are simple and well-established methods

TABLE 3 Mortality following acute exacerbation

Mortality rate ${ }^{\pi} \quad$ Patients with investigator-reported acute exacerbations

\section{Patients with events adjudicated as confirmed or suspected acute exacerbations ${ }^{\#}$}

Patients with all events adjudicated as not acute exacerbations"

\begin{tabular}{|c|c|c|c|c|c|c|}
\hline & & \multirow{2}{*}{\multicolumn{2}{|c|}{ Nintedanib (n=14) }} & & \\
\hline & Nintedanib $(n=34)$ & Placebo $(n=35)$ & & & Nintedanib (n=23) & Placebo (n=13) \\
\hline 90-day & $10(29.4)$ & $15(42.9)$ & 5 (35.7) & $12(48.0)$ & $6(26.1)$ & 7 (53.9) \\
\hline
\end{tabular}

\#: analyses based on patients who had one or more adjudicated confirmed or suspected acute exacerbation or who had one or more event that was adjudicated and all events were adjudicated as not acute exacerbations; ${ }^{\text {: }}$ : calculated as the number (\%) of patients who died within 30,90 or 180 days of onset of a first exacerbation event. The three categories of patient shown in this table are not mutually exclusive. 
to identify covariates that improve the performance of a statistical model. However, these methods have limitations, including the potential for bias and overfitting, which have been discussed in the literature previously [24], and alternative selection methods such as least absolute shrinkage and selection operator or cross-validation could have been used.

In conclusion, we present analyses from the INPULSIS dataset demonstrating that acute respiratory events, identified as acute exacerbations of IPF by local site investigators were associated with poor outcomes, regardless of whether they were centrally adjudicated as confirmed or suspected acute exacerbations, or as some other type of respiratory worsening. Additional findings from our analyses support the concept that acute exacerbation of IPF is more common in patients with more physiologically advanced disease and that treatment with nintedanib may reduce the risk of developing an acute exacerbation. While our data suggest nintedanib may improve post-acute exacerbation survival, these data are exploratory and require validation in more robust cohorts. Future research into acute exacerbation risk and the impact of approved therapies on this risk, ideally with pooling of primary datasets from multiple clinical trials, is greatly needed.

\section{Acknowledgements}

The INPULSIS trials were funded by Boehringer Ingelheim. Editorial assistance, supported financially by Boehringer Ingelheim, was provided by Julie Fleming and Wendy Morris of Fleishman-Hillard Group, Ltd, London, UK, during the preparation of this article. The authors (except M. Luisetti) were fully responsible for all content and editorial decisions, were involved at all stages of manuscript development, and approved the final version.

\section{References}

1 Raghu G, Collard HR, Egan JJ, et al. An official ATS/ERS/JRS/ALAT statement: idiopathic pulmonary fibrosis: evidence-based guidelines for diagnosis and management. Am J Respir Crit Care Med 2011; 183: 788-824.

2 Ley B, Collard HR, King TE Jr. Clinical course and prediction of survival in idiopathic pulmonary fibrosis. Am J Respir Crit Care Med 2011; 183: 431-440.

3 Collard HR, Moore BB, Flaherty KR, et al. Acute exacerbations of idiopathic pulmonary fibrosis. Am J Respir Crit Care Med 2007; 176: 636-643.

4 Kondoh Y, Taniguchi H, Katsuta $\mathrm{T}$, et al. Risk factors of acute exacerbation of idiopathic pulmonary fibrosis. Sarcoidosis Vasc Diffuse Lung Dis 2010; 27: 103-110.

5 Song JW, Hong SB, Lim CM, et al. Acute exacerbation of idiopathic pulmonary fibrosis: incidence, risk factors and outcome. Eur Respir J 2011; 37: 356-363.

6 Collard HR, Yow E, Richeldi L, et al. Suspected acute exacerbation of idiopathic pulmonary fibrosis as an outcome measure in clinical trials. Respir Res 2013; 14: 73.

7 Kondoh Y, Taniguchi H, Ebina M, et al. Risk factors for acute exacerbation of idiopathic pulmonary fibrosis extended analysis of pirfenidone trial in Japan. Respir Investig 2015; 53: 271-278.

8 Costabel U, Inoue Y, Richeldi L, et al. Efficacy of nintedanib in idiopathic pulmonary fibrosis across pre-specified subgroups in INPULSIS. Am J Respir Crit Care Med 2016; 193: 178-185.

9 Akira M, Kozuka T, Yamamoto S, et al. Computed tomography findings in acute exacerbation of idiopathic pulmonary fibrosis. Am J Respir Crit Care Med 2008; 178: 372-378.

10 Lee JS, Song JW, Wolters PJ, et al. Bronchoalveolar lavage pepsin in acute exacerbation of idiopathic pulmonary fibrosis. Eur Respir J 2012; 39: 352-358.

11 Lee JS, Collard HR, Anstrom KJ, et al. Anti-acid treatment and disease progression in idiopathic pulmonary fibrosis: an analysis of data from three randomised controlled trials. Lancet Respir Med 2013; 1: 369-376.

12 Ohshimo S, Ishikawa N, Horimasu Y, et al. Baseline KL-6 predicts increased risk for acute exacerbation of idiopathic pulmonary fibrosis. Respir Med 2014; 108: 1031-1039.

13 Johannson KA, Vittinghoff $\mathrm{E}$, Lee $\mathrm{K}$, et al. Acute exacerbation of idiopathic pulmonary fibrosis associated with air pollution exposure. Eur Respir J 2014; 43: 1124-1131.

14 Yu YF, Macaulay DS, Reichmann WM, et al. Association of early suspected acute exacerbations of idiopathic pulmonary fibrosis with subsequent clinical outcomes and healthcare resource utilization. Respir Med 2015; 109: 1582-1588.

15 Wollin L, Wex E, Pautsch A, et al. Mode of action of nintedanib in the treatment of idiopathic pulmonary fibrosis. Eur Respir J 2015; 45: 1434-1445.

16 Richeldi L, Costabel U, Selman M, et al. Efficacy of a tyrosine kinase inhibitor in idiopathic pulmonary fibrosis. N Engl J Med 2011; 365: 1079-1087.

17 Richeldi L, du Bois RM, Raghu G, et al. Efficacy and safety of nintedanib in idiopathic pulmonary fibrosis. $N$ Engl J Med 2014; 370: 2071-2082.

18 Ohshimo S, Ishikawa N, Horimasu Y, et al. Ethnic differences in the incidence of acute exacerbation in idiopathic pulmonary fibrosis. Am J Respir Crit Care Med 2013; 187: A4345.

19 Natsuizaka M, Chiba H, Kuronuma K, et al. Epidemiologic survey of Japanese patients with idiopathic pulmonary fibrosis and investigation of ethnic differences. Am J Respir Crit Care Med 2014; 190: 773-779.

20 Taniguchi H, Ebina M, Kondoh Y, et al. Pirfenidone in idiopathic pulmonary fibrosis. Eur Respir J 2010; 35: 821-829.

21 Noth I, Anstrom KJ, Calvert SB, et al. A placebo-controlled randomized trial of warfarin in idiopathic pulmonary fibrosis. Am J Respir Crit Care Med 2012; 186: 88-95.

22 de Andrade J, Schwarz M, Collard HR, et al. The Idiopathic Pulmonary Fibrosis Clinical Research Network (IPFnet): diagnostic and adjudication processes. Chest 2015; 148: 1034-1042.

23 Collard HR, Ryerson CJ, Corte TJ, et al. Acute exacerbation of idiopathic pulmonary fibrosis. An international working group report. Am J Respir Crit Care Med 2016; 194: 265-275.

24 Harrell F. Regression Modeling Strategies: With Applications to Linear Models, Logistic Regression, and Survival Analysis. 1st Edn. New York, Springer-Verlag, 2001. 